\title{
COMPUTATIONAL DESIGN OF NOVEL ORAL INSULIN CONJUGATES FOR THE DEVELOPMENT OF SOLID ORAL INSULIN DOSAGE FORMS
}

\author{
JAYARAMA REDDY* \\ Department of Botany, St. Joseph College, Bengaluru, Karnataka, India. Email: drjayaramreddy@gmail.com
}

Received: 12 February 2020, Revised and Accepted: 21 March 2020

ABSTRACT

Objective: Insulin is used to manage diabetes mellitus by subcutaneous injections regulary, that has become a part of patients life and is distressing for patients. Hence, we anticipate to get rid of the usage of subcutaneous injections completely from the medicine world and would make the diabetic people to say as "farewell to painful injections."

Methods: Virtual screening method (for selection of carriers), Toxtree tool (for toxicity evaluation of carriers), and discovery studio tool (for pharmacophore designing, absorption, distribution, metabolism, excretion, and toxicity [ADMET] analysis, designing oral insulin conjugates (OICs), and interaction studies between insulin receptor and OICs) were used for proposed study.

Results: We have screened 14 competent drug delivering molecules (DDMs) from seven chemical compound databases. The ADMET and pharmacophoric properties of DDMs were analyzed by drug-informatics' tools. Consequently, the DDMs were mono, di, and poly conjugated by covalent bonding with various binding sites of monomeric and hexameric form of human insulin and insulin-lispro (Humalogß) individually; and novel OICs were generated. Its binding efficiency and biological activity with insulin-receptor were determined.

Conclusion: Inulin and Vitamin-B1 are the novel, safe, and proficient carriers for oral delivery of insulin. Insulin lispro is the remarkable option for oral delivery than other insulin forms.

Keywords: Oral insulin: Diabetes, Drug delivery, Insulin tablets.

(c) 2020 The Authors. Published by Innovare Academic Sciences Pvt Ltd. This is an open access article under the CC BY license (http://creativecommons. org/licenses/by/4. 0/) DOI: http://dx.doi.org/10.22159/ajpcr.2020.v13i5.37308

\section{INTRODUCTION}

Drug discovery is an expensive but essential process which leads to innovation of therapeutic molecules to manage or cure diverse ailments of human beings through various modes of administration [1]. Some peculiar drugs such as proteins, hormones, enzymes, and peptides require efficient drug delivering molecules (DDMs) especially for oral formulations to reach therapeutic targets against the interruption of biological barriers. Hence, as like drug discovery process, discovering DDMs to designing oral protein conjugates is the much decisive procedure to overcome the biological barriers persists in administration of protein as oral tablet formulation [2]. In addition, in the absence of DDMs, the time and cost for the discovery process of some novel drugs will goes in vain and the dream of oral protein tablet formulations would become zero. In this perspective, we are positioning the DDMs, just one step higher than drugs; anyway that is the truth also. Compared to drugs, the DDMs should possess improved physicochemical properties and nil biological activity to carry and make the drugs to reach the target and produce desired effects. Nowadays, numerous protein and hormone drugs are administered through various modes of painful injections which are making "fear of pain" among patients [3]. In this case, the subcutaneous injection of insulin formulations has been the core therapy utilized for the management of the major global health threat of type 1 diabetes as well as type 2 diabetes since insulin's discovery over 85 years ago [2]. There are numerous disadvantages of this subcutaneous administration such as poor compliance, local discomfort, inconvenience, fright of soreness of multiple injections, hypoglycemic risk related to injections, occasional hyper-insulinemia due to overdoses, unsatisfactory metabolic regulations, allergy, harrowing, and insulin lipodystrophy at the site of injection [3]. In addition, subcutaneously administered insulin is absorbed directly into the peripheral circulation without initial hepatic circulation, thereby exposing peripheral targets to higher insulin concentrations relative to the liver [3].
In contrast to subcutaneous injection, oral insulin conjugates (OICs) has the potential to mimic the natural route of endogenous insulin secreted by the pancreas through the portal vein directly to the liver and then to the peripheral circulation, resulting in a portal-peripheral insulin gradient. Hence, oral insulin is more convenient, compliance, and physiologically desirable among diabetic patients. The oral delivery of insulin still has many problems mainly due to natural characteristics of insulin such as physicochemical instability, increased susceptibility to gastric digestion and proteolysis, low solubility, higher molecular weight (5808 Da), and poor permeability to across the intestinal epithelial membrane [4]. To overcome these barriers, the earlier studies have been proposed very few number of chemically-modified insulin such as insulin-deoxycholic acid chemical conjugates [5], poly(ethylene glycol)-insulin conjugates, methoxy-poly(ethylene glycol)-insulin conjugates (IN-105), hexylinsulin monoconjugate-2, insulin-transferrin conjugates [6], hyaluronaninsulin complex, and Vitamin B12-insulin complex. These OICs possess the basic construction of DDM-insulin complexes and showed improved therapeutic profile such as gastric and enzymatic stability, solubility, circulation half-life, or permeability against mucosal membrane [4]. OICs from all of these studies significantly protected the bound proteins from digestion, as well as facilitating their transport into blood serum in Phase I. However, these researches were not successfully complete in the Phase II trial. As of 2014, no products appeared to be successful in the market and not even in the III-phase of the clinical trial because of lack of physical stability against biological barriers [7]. The proposed analysis could be successful in identification of more efficient drug delivering agents which would help the insulin molecule to pass through the Phase III clinical trial then to the market.

In our analysis, the monomeric and hexameric form of recombinant human insulin and insulin-lispro (Humalog ${ }^{\circledR}$ ) was modified by covalent bonding of short-chain DDMs to the amino-acids of PheB1 and LysB29 in recombinant human insulin, and also PheB1 and LysB28 in insulin-lispro. Because, the 
conjugation of DDMs through covalent bonding to the PheB1 $\left(\mathrm{N}^{\alpha \mathrm{B} 1}\right)$ or LysB29 $\left(\mathrm{N}^{\beta B 29}\right)$ amino groups of insulin ought to have no negative impact on the insulin's therapeutic activity since it has been formerly determined that these residues do not directly contribute in receptor binding [8]. On these perspectives, a study proven that DDM-human crystalline Zn-insulin conjugate (at PheB1) was slightly stable against acidic and enzymatic degradation, and also resistance to fibrillation in aqueous solution when correlated with commercially available subcutaneous injection $\left(\right.$ Humulin $\left.^{\circledast}\right)$. In this case, DDM-human crystalline Zn-insulin conjugate (at LysB29) was physically more stable than DDM-human crystalline Zn-insulin conjugate (at PheB1) and Humulin ${ }^{\circledR}$. In addition, these DDMhuman crystalline $\mathrm{Zn}$-insulin conjugates were more resistant to digestive activity of trypsin and chymo-trypsin than human native-insulin. Hence, OICs effectively inhibits insulin-fibrillation in aqueous solution and improve its stability against temperature, $\mathrm{pH}$, and interfacial and shear forces [9]. Insoluble insulin-fibrils are responsible for immunogenicity, antigenicity, and not for biological activity. In another study, DDM-human crystalline Zn-insulin conjugate (at PheB1) was found as less immunogenic and antigenic than human native-insulin [10]. The conjugation of insulin with DDM can protect the self-aggregation (dimerization) in aqueous solutions. In this stance, insulin-lispro has higher resistant capability against self-aggregation than human recombinant and native-insulin. The oligomeric form (chiefly hexamers) of insulin is not bioactive and the fraction of amount is absorbed across the capillary endothelium into the systemic circulation in the absence of DDM. The dissociation of oligomer into dimmers and monomers is seen as the rate limiting barrier to absorption that effectively affects the preparation's pharmacological response. In a study, the in vivo pharmacodynamic assay reveals that there is no loss in biological activity after conjugation of carrier to the either site on the oligomeric form of insulin-B-chain. The attachment of long-chain DDM (2000 Da) decreased the bioactivity of conjugates than sort-chain DDM (750 Da). Hence, mono-disperse and short-chain DDMs ( $\leq 750 \mathrm{Da})$ are preferred. The experimental findings support that the concept of improving oligomeric insulin's pharmacokinetic and pharmacodynamic properties through novel DDMs by conjugation method. We report and analyze the structural series of novel DDMs and OICs by drug-informatics.

\section{METHODS}

\section{Carriers}

Screening

For virtual screening of DDMs, PubChem Compound, Zinc Database, Kyoto Encyclopedia of Genes and Genomes, DrugBank, ChemSpider, ChEMBL, and Chemical Entities of Biological Interest were used. Carrier compounds were retrieved by the search terms of "Polymer" and "Biopolymer." The compounds were filtered through literature analysis from the previous studies and virtual screening using a defined criteria listed below, in part akin to the Lipinski's rule [11]; the DDMs should be: (a) Mono-disperse, (b) short-chain (low-molecular weight [<2000 Da]), (c) biocompatible, (d) lipophilic, (e) physically stability against gastric acids and proteolytic enzymes, (f) inert (no biological activity), and (g) non-toxic.

\section{Analysis of pharmacophoric features}

The retrieved compounds were subjected for pharmacophore analysis by Discovery Studio (Accelarys discovery studio 2.5) [12]. A pharmacophore model, according to the IUPAC definition, is "an ensemble of steric and electronic features that is necessary to ensure the optimal intermolecular interactions with a specific biological target and to trigger (or block) its biological response." In discovery studio, a pharmacophore is defined as the essential features or chemical substructures and their corresponding 3D locations that are responsible for the similar biological activities of a set of compounds. Typically, pharmacophore features include hydrophobic (in light blue), hydrogen bond acceptor (HBA, in green), hydrogen bond donor (HBD, in Magenta), and active principles.

\section{Analysis of physicochemical properties}

The retrieved compounds were subjected for absorption, distribution, metabolism, excretion, and toxicity (ADMET) evaluation by Discovery Studio. ADMET Descriptors include:
- Aqueous solubility: This model uses linear regression to predict the solubility of each compound in water at $25^{\circ} \mathrm{C}$. Key to aqueous solubility levels:

\begin{tabular}{lll}
\hline Level & Value & Drug-likeness \\
\hline 0 & $\log (\mathrm{Sw})<-8.0$ & Extremely low \\
1 & $-8.0<\log (\mathrm{Sw})<-6.0$ & No, very low, but possible \\
2 & $-6.0<\log (\mathrm{Sw})<-4.0$ & Yes, low \\
3 & $-4.0<\log (\mathrm{Sw})<-2.0$ & Yes, good \\
4 & $-2.0<\log (\mathrm{Sw}) 0.0==^{\prime \prime \prime}$ & Yes, optimal \\
5 & $0.0<\log (\mathrm{Sw})$ & No, too soluble \\
\hline
\end{tabular}

$\log (\mathrm{Sw})$ : Log aqueous solubility (solubility in water)

- Blood-brain barrier (BBB) penetration: This model predicts bloodbrain penetration (BBB) after oral administration. This model contains a quantitative linear regression model for the prediction of blood-brain penetration, as well as $95 \%$ and $99 \%$ confidence ellipses in the ADMET_PSA_2D, ADMET_AlogP98 plane (ADMET_PSA_2D: Fast polar surface area; and ADMET_AlogP98: Atom-based LogP). There are four prediction levels within the $95 \%$ and $99 \%$ confidence ellipsoids; no prediction is made for compounds outside the $95 \%$ and $99 \%$ confidence ellipsoids (undefined level $=4$ ).

\begin{tabular}{|c|c|c|}
\hline Level & Value & Description \\
\hline 0 & $\begin{array}{l}\text { Very high penetrants } \\
(\log B B \geq 0.7)\end{array}$ & Brain-blood ratio $>5: 1$ \\
\hline 1 & $\begin{array}{l}\text { High penetrants } \\
(0 \leq \log B B<0.7)\end{array}$ & $\begin{array}{l}\text { Brain-blood ratio } \\
\text { between 1:1 and 5:1 }\end{array}$ \\
\hline 2 & $\begin{array}{l}\text { Medium penetrants } \\
(-0.52<\log B B<0)\end{array}$ & $\begin{array}{l}\text { Brain-blood ratio } \\
\text { between } 0.3: 1 \text { and 1:1 }\end{array}$ \\
\hline 3 & $\begin{array}{l}\text { Low penetrants } \\
(\operatorname{logBB} \leq-0.52)\end{array}$ & $\begin{array}{l}\text { Brain-blood } \\
\text { ratio }<0.3: 1\end{array}$ \\
\hline 4 & Undefined & $\begin{array}{l}\text { Outside 99\% } \\
\text { confidence ellipse }\end{array}$ \\
\hline
\end{tabular}

logBB: Log brain-blood

- CYP2D6 binding: Predicts cytochrome P450 2D6 enzyme inhibition. The cytochrome P450 2D6 model predicts CYP2D6 enzyme inhibition using 2D chemical structure as input. The model classifies compounds as either 0 or 1 for non-inhibitor or inhibitor and provides an average class value estimate of confidence. Key to CYP2D6;

\begin{tabular}{lll}
\hline $\begin{array}{l}\text { Predicted } \\
\text { class }\end{array}$ & Value & Description \\
\hline 0 & Non-inhibitor & $\begin{array}{l}\text { Unlikely to inhibit CYP2D6 enzyme; } \\
\text { ADMET_CYP2D6_Probability<0.5 } \\
\text { Likely to inhibit CYP2D6 enzyme; } \\
\text { ADMET_CYP2D6_Probability>0.5 }\end{array}$ \\
\hline & Inhibitor & \\
\hline $\begin{array}{l}\text { ADMET_CYP2D6: Predicted class ADMET_CYP2D6_Probability: CYP2D6 score } \\
\text { or average class value }\end{array}$
\end{tabular}

- Hepatotoxicity: Predicts the occurrence of dose-dependent human hepatotoxicity. The hepatotoxicity model predicts potential organ toxicity for a wide range of structurally diverse compounds. Key to hepatotoxicity;

\begin{tabular}{lll}
\hline $\begin{array}{l}\text { Predicted } \\
\text { class }\end{array}$ & Value & Description \\
\hline 0 & Nontoxic & $\begin{array}{l}\text { Unlikely to cause dose-dependent } \\
\text { liver injuries. ADMET_- }\end{array}$ \\
& & $\begin{array}{l}\text { Hepatotoxicity_Probability }<0.5 \\
\text { Likely to cause dose-dependent } \\
\text { liver injuries. ADMET__ } \\
\text { Hepatotoxicity_Probability }>0.5\end{array}$ \\
\hline
\end{tabular}

ADMET_Hepatotoxicity: Predicted class ADMET_Hepatotoxicity_Probability: Hepatotoxicity score (average class value) 
- Intestinal absorption: This model predicts human intestinal absorption after oral administration. Intestinal absorption is defined as a percentage absorbed rather than as a ratio of concentrations (cf. blood-brain penetration). A well-absorbed compound is one that is absorbed at least $90 \%$ into the bloodstream in humans. The intestinal absorption model includes 95\% and 99\% confidence ellipses in the ADMET_PSA_2D, ADMET_AlogP98 plane. The ellipses define regions where well-absorbed compounds are expected to be found: $95 \%$ of well-absorbed compounds are expected to fall within the $95 \%$ ellipse, while $99 \%$ of well-absorbed compounds should fall within the $99 \%$ ellipse. Note that, the location of any particular compound does not necessarily imply whether it will be well, moderately, or poorly absorbed. In general, however, absorption tends to drop off quite rapidly outside the 95\% ellipse. These levels are defined by the $95 \%$ (blue line) and $99 \%$ (magenta line) confidence ellipsoids. There are four prediction levels:

\begin{tabular}{lll}
\hline Level & Value & Description \\
\hline 0 & $\begin{array}{l}\text { ADMET_Absorption_T2_2D }<6.1261 \\
\text { (inside 95\%) }\end{array}$ & Good absorption \\
& $\begin{array}{l}\text { 6.1261 } \leq \text { ADMET_Absorption_ } \\
\text { T2_2D<9.6026 (inside 99\%) }\end{array}$ & Moderate absorption \\
& 9.6026<ADMET_Absorption_ & Low absorption \\
2 & T2_2D (outside 99\%) & Very low absorption \\
3 & ADMET_PSA_2D $\geq 150.0$ or & \\
& ADMET_AlogP98 $\leq-2.0$ or ADMET_ & \\
& AlogP98 $\geq 7.0$ &
\end{tabular}

ADMET_PSA_2D: Fast polar surface area ADMET_AlogP98: ALogP ADMET_ Absorption_T2_2D is the Mahalanobis distance for the compound in the ADMET_PSA_2D, ADMET_AlogP98 plane. It is referenced from the center of the region of chemical space defined by well absorbed compounds

- Plasma protein binding: The plasma protein binding model predicts whether a compound is likely to be highly bound to carrier proteins in the blood. Key to plasma protein binding;

\begin{tabular}{ll}
\hline Level & Description \\
\hline 0 & Binding is $<90 \%$ (no markers flagged and AlogP98<4.0) \\
1 & Binding is $>90 \%$ (flagged at $90 \%$ or AlogP98 $>4.0$ ) \\
2 & Binding is $>95 \%$ (flagged at $95 \%$ or AlogP98 $>5.0$ ) \\
\hline AlogP98: Atom-based LogP from FastDesc
\end{tabular}

\section{Analysis of toxicity}

The retrieved compounds were subjected for toxicity evaluation by discovery studio and TOXTREE (by IdeaConsult Ltd.(Sofia, Bulgaria) [13]. Toxtree is able to estimate toxic hazard by applying a decision tree approach. The classification result is shown in graphical form (green highlight for Class I [non-toxic], yellow highlight for Class II [moderately toxic], and red highlight for Class III [Toxic]), as well as in text form. In discovery studio; Toxicity Prediction by Komputer Assisted Technology (TOPKAT) models have been re-trained using updated training sets from the legacy TOPKAT. The following models are extensible and are derived using calculable properties [14];

- FDA rodent carcinogenicity

- Ames mutagenicity

- Rat oral LD $\mathrm{LD}_{50}$

- Rat maximum tolerated dose (MTD)

- Skin irritancy

- Skin sensitization

- Aerobic biodegradability

\section{Designing OICs and OIC - insulin receptor (IR) binding}

For designing OICs and interaction of OIC with IR were carried out through "LibDock" algorithm of discovery studio $[13,15]$. The LibDock docking program performs the following steps using a set of pre-generated ligand conformations and a receptor with a specified binding site:
- $\quad$ Remove hydrogen atoms

- Rank ligand conformations and prune by solvent accessible solvent area

- $\quad$ Find hotspots using a grid placed into the binding site and using polar and apolar probes. The numbers of hotspots are pruned by clustering to a user defined value

- Dock ligand poses by aligning to the hotspots. This is performed by using triplets (i.e., three ligand atoms are aligned to three receptor hotspots). Poses which result in protein clashes are removed

- A final broyden-fletcher-goldfarb-shanno pose optimization stage is performed using a simple pair-wise score (similar to piecewise linear potential). The top scoring ligand poses are retained

- Hydrogen atoms are added.

Hydrogen atoms added in the final step may result in small bumps with the protein. Therefore, minimization should be performed before using scoring functions that are sensitive to such bumps.

\section{RESULTS}

Carriers

Screening

In carrier screening, more than 1,00,000 compounds were retrieved from seven compound databases [15]. Among those, 14 compounds were screened using experimental text mining and filtration criteria (Table 1). According to the data mining, most of the screened compounds in Table 1 are monodisperse such as Vitamin B12, Vitamin H, folic acid, poly-N-vinylpyrrolidone, inulin, poly cysteine, chitosan, pectin, poly (propylene glycol), poly (propylene imine), poly (lactic-co-glycolic acid), deoxycholic acid except Vitamin B1, and L-carnitine, because of the lack of experimental data. Molecular weight of polymeric DDMs is varies based on length of chain, but in the case of vitamins, molecular weights are measurable. Maximum carriers in the retrieved list has shown low-molecular weight due to short-chain in structure $(<2000 \mathrm{Da}$. L-Carnitine (162.113 Da), poly-N-vinylpyrrolidone (11.141 Da), inulin (342.297 Da), poly cysteine (121.158 Da), pectin (194.139 Da), and poly (propylene glycol) (76.094 Da) are possess low-molecular weight while compare with vitamins and other macromolecules in the list. All the listed molecules are biocompatible and biodegradable.

Most of the carriers are having efficient oral bioavailability and intestinal permeability such as Vitamin B12, Vitamin H, folic acid, Vitamin B1, L-carnitine, Inulin, poly (propylene glycol), poly (propylene imine), poly (lactic-co-glycolic acid), and deoxycholic acid. Chitosan and pectin are graded as moderately efficient in bioavailability while poly$\mathrm{N}$-vinylpyrrolidone and poly cysteine are very poor intestinal transport, because of the lack of lipophilicity. Biomedical text mining shows that all the listed compounds are physically stable against gastric acids and proteolytic enzymes during drug delivery. Among the retrieved carriers, Vitamin B12, Vitamin H, Vitamin B1, poly-N-vinylpyrrolidone, inulin, chitosan, poly (propylene glycol), poly (lactic-co-glycolic acid), and deoxycholic acid are chemically inert and they does not undergoes any biochemical transformation and aggregation during drug delivery. Through text-mining, we could not found whether folic acid, L-Carnitine, poly cysteine, pectin, and poly (propylene imine) are inert or not. Based on the concept of toxicity, Vitamin B12, Vitamin H, folic acid, Vitamin B1, L-carnitine, inulin, poly cysteine, chitosan, pectin, and poly (lacticco-glycolic acid) are non-toxic materials and do not produce any untoward reactivity during drug-delivery mechanism. However, poly$\mathrm{N}$-vinylpyrrolidone, poly (propylene glycol), poly (propylene imine), and deoxycholic acid are moderately toxic based on text-mining.

The experimental text-mining concludes inulin, chitosan and poly (lactic-co-glycolic acid) are efficient, safe and primary DDMs of drugs that completely fulfill the filtration criteria. Inulin reduces the production of potentially toxic metabolites, induce important immunemediated effects and reduce the cancer risk during drug delivery. In general inulin and chitosan are participate in colon-targeting drug delivery, possess very minimum exposure to gastric fluids in stomach 
and enzymatic degradation in small intestine. Folic acid, L-Carnitine, and pectin are eligible for drug delivery process, but we could not conclude whether they are inert. Vitamin B1 is an effectual and nontoxic carrier, may cause anaphylaxis by higher doses [18]. L-Carnitine and Vitamin B1 do not hold any literature evidence that they possess mono-disperse character or not. Vitamin B12 is inert, long-term usage may cause Vitamin-B12 deficiency. Vitamin $\mathrm{H}$ is inert, but it may inert the biological activity of drug. Poly-N-vinylpyrrolidone and poly (propylene imine) are participating in nanoparticle based drug delivery, and both are moderately toxic. Poly cysteine is hydrophilic in nature; shows poor permeability across intestinal epithelium; and no research confirmation whether it is inert. Poly(propylene glycol) is an efficient carrier for drug delivery, and inert, overdose may cause skin and eye irritation. It may induce cardiotoxic effects include arrhythmias and cardiac arrest, and central nervous system depression, renal and hepatic damage has also reported. In case studies, toxic symptoms appeared only after frequent doses of propylene glycol, used as a vehicle in medicines, were repetitively applied to the skin. In drug delivering mechanism, it eliminates toxic degradation. It is less toxic than the parent substance (poly ethylene glycol). Deoxycholic acid is a prominent carrier, which is not inert and moderately toxic; may promote colon tumorigenesis in both animals and humans.
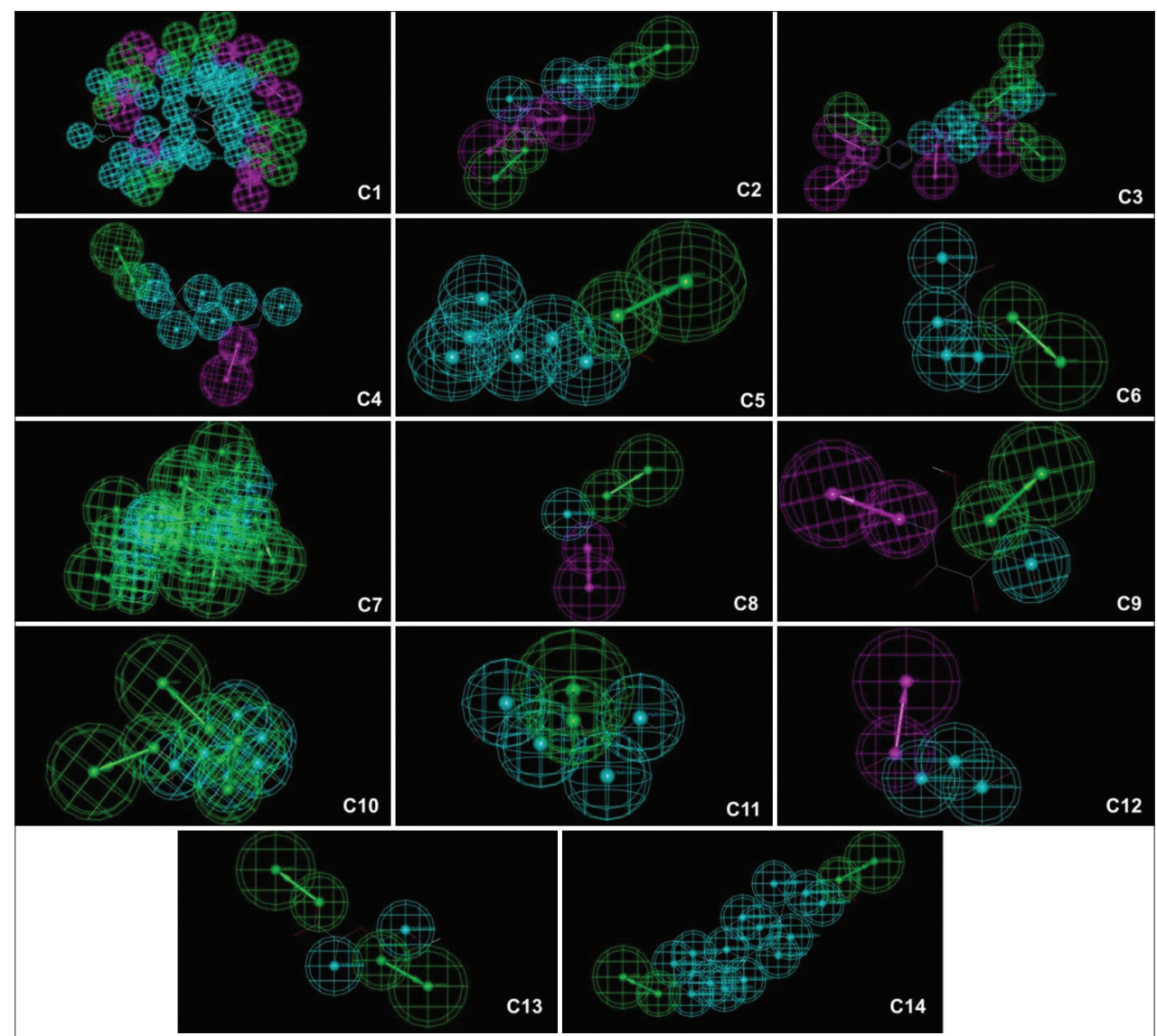

Fig. 1: Pharmacophoric features of drug delivering molecules (DDMs) are illustrated by discovery studio software. Acceptors (green in color), donors (magenta in color), and hydrophobic regions (blue in color) of pharmacophoric features of all DDMs are demonstrated and differentiated by color: C1 - Vitamin B12 (cobalamin); C2 - Vitamin H (Biotin); C3 - folic acid (Vitamin M/Vitamin B9); C4 - Vitamin B1 (Thiamin); C5 - L-Carnitine (Vitamin BT); C6 - Poly-N-vinylpyrrolidone; C7; Inulin; C8 - poly cysteine; C9 - Chitosan; C10 - Pectin; C11 Poly(propylene glycol); C12 - Poly(propylene imine); C13 - Poly (lactic-co-glycolic acid); and C14 - Deoxycholic acid

Table 2: Pharmacophoric features of carriers

\begin{tabular}{llllll}
\hline $\begin{array}{l}\text { Carrier } \\
\text { No }\end{array}$ & Carrier & $\begin{array}{l}\text { Number of acceptor } \\
\text { in molecule }\end{array}$ & $\begin{array}{l}\text { Number of donors } \\
\text { in molecule }\end{array}$ & $\begin{array}{l}\text { Number of } \\
\text { hydrophobic region }\end{array}$ & $\begin{array}{l}\text { Number of } \\
\text { pharmacophore principle }\end{array}$ \\
\hline C1 & Vitamin B12 (cobalamin) & 8 & 7 & 29 & Nil \\
C2 & Vitamin H (Biotin) & 2 & 2 & 5 & 6 \\
C3 & Folic acid (Vitamin M/Vitamin B9) & 4 & 4 & 7 & 10 \\
C4 & Vitamin B1 (Thiamin) & 1 & 1 & 7 & 10 \\
C5 & L-Carnitine (Vitamin BT) & 1 & 0 & 6 & 2 \\
C6 & Poly-N-vinylpyrrolidone & 1 & 0 & 4 & Nil \\
C7 & Inulin & 11 & 0 & 12 & 7 \\
C8 & Poly cysteine & 1 & 1 & 1 & 10 \\
C9 & Chitosan & 1 & 1 & 1 & 10 \\
C10 & Pectin & 3 & 0 & 6 & 4 \\
C11 & Poly (propylene glycol) & 1 & 0 & 4 & Nil \\
C12 & Poly (propylene imine) & 0 & 1 & 3 & 8 \\
C13 & Poly (lactic-co-glycolic acid) & 2 & 0 & 2 & 7 \\
C14 & Deoxycholic acid & 2 & 0 & 14 & \\
\hline
\end{tabular}


Analysis of pharmacophoric features

Pharmacophores are conceptual description of molecular principles or features that are essential for molecular recognition of a molecule through a biological macromolecule. Pharmacophoric features of DDMs are illustrated and demonstrated by Discovery Studio software (Table 2; Fig. 1). Pharmacophore features comprise HBD, HBA, hydrophobic centroids, aromatic rings, cations, and anions. Among those features, acceptor, donor, hydrophobic regions, and number of active principles of carrier molecules (Fig. 1) were investigated based on Lipinski's rule. According to the rule of Lipinski, a molecule should not donate more than 5 hydrogen bonds and it should not accept more than 10 hydrogen bonds. Most of the carriers obey the rule and eligible for drug delivery except Inulin and Vitamin B12. Inulin possesses 11 HBA ( $\leq 10$ as per rule) may leads to accept electrons transferred to it from another compound. Inulin may be an oxidizing molecule, by virtue of its accepting electrons, it itself reduced in the drug delivery process. Inulin may undergo permanent chemical alteration through covalent or ionic reaction chemistry, resulting in the complete and irreversible transfer of one or more electrons. However, in this case, inulin is a colon targeting molecule; subsequently, it does not undergo any chemical transformation during drug delivery mechanism like Chitosan. Vitamin B12 possesses 7 HBD $(\leq 5$ as per rule) may leads to donate electrons to another compound. Vitamin B12 may be a reducing agent, by virtue of its donating electrons, it itself oxidized in the drug
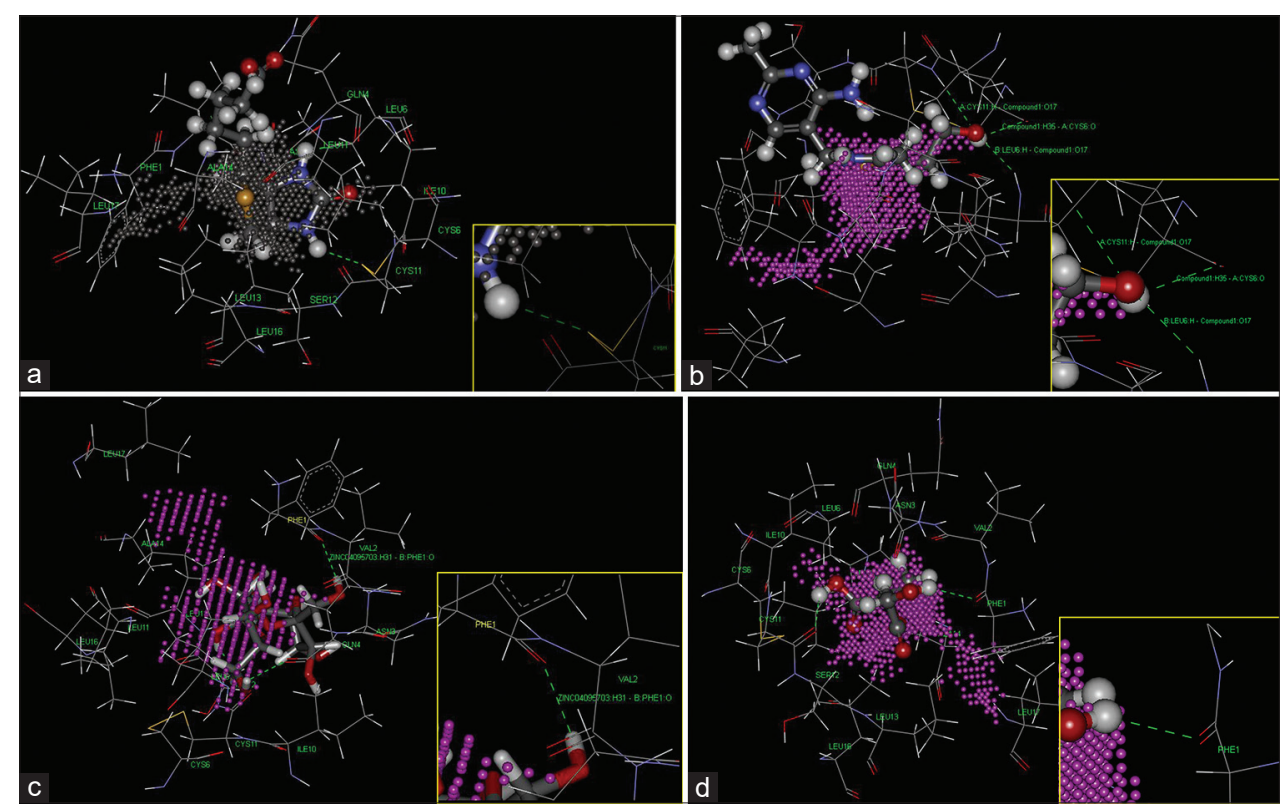

Fig. 2: (a) Bioconjugate from human insulin monomer protein data bank (PDB ID: 1HLS) and Vitamin H is illustrated by discovery studio software. Vitamin H conjugated at CYS A11, GLN B4, and HIS B10 amino acids of insulin monomer. The inner figure illustrates the CYS 11 amino acid of insulin-A chain conjugates with Vitamin H. (b) Bioconjugate from human insulin monomer (PDB ID: 1HLS) and Vitamin B1 is illustrated by discovery studio software. Vitamin B1 conjugated at CYS A6, CYS A11, and LEU B6 amino acids of insulin monomer.

The inner figure illustrates the CYS 6, CYS 11 amino acids of insulin-A chain, and LEU 6 amino acid of insulin-B chain conjugates with Vitamin B1. (c) Bioconjugate from human insulin monomer (PDB ID: 1HLS) and inulin is illustrated by discovery studio software. Inulin conjugated at PHE B1 amino acid of insulin monomer. The inner figure illustrates the PHE 1 amino acid of insulin-B chain conjugates with inulin. (d) Bioconjugate from human insulin monomer (PDB ID: 1HLS) and poly (lactic-co-glycolic acid) is illustrated by discovery studio software. Poly (lactic-co-glycolic acid) conjugated at PHE B1 and CYS A11 amino acid of insulin monomer. The inner figure illustrates the PHE 1 amino acid of insulin-B chain conjugates and with poly (lactic-co-glycolic acid)

Table 3: Physicochemical properties of drug delivering molecules

\begin{tabular}{|c|c|c|c|c|c|c|c|}
\hline \multirow[t]{2}{*}{ S. No. } & \multirow[t]{2}{*}{ Carriers } & \multicolumn{6}{|c|}{ ADME descriptors } \\
\hline & & $\begin{array}{l}\text { Aqueous } \\
\text { solubility }\end{array}$ & $\begin{array}{l}\text { Blood brain barrier } \\
\text { penetration }\end{array}$ & CYP 2D6 Binding & Hepatotoxicity & Intestinal absorption & Plasma protein binding \\
\hline 2 & $\mathrm{C} 2$ & -1.432 & -1.229 & 0.069 & 0.264 & 0.0 & 0.0 \\
\hline 3 & $\mathrm{C} 3$ & -3.378 & 4.0 & 0.277 & 0.662 & 3.0 & 0.0 \\
\hline 4 & $\mathrm{C} 4$ & -1.335 & -1.253 & 0.336 & 0.423 & 0.0 & 0.0 \\
\hline 5 & $\mathrm{C} 5$ & 2.734 & 4.0 & 0.059 & 0.066 & 3.0 & 0.0 \\
\hline 6 & C6 & -0.550 & 4.0 & 0.059 & 0.324 & 1.0 & 0.0 \\
\hline 7 & $\mathrm{C} 7$ & 0.894 & 4.0 & 0.108 & 0.0139 & 3.0 & 0.0 \\
\hline 8 & $\mathrm{C} 8$ & 0.335 & -1.362 & 0.059 & 0.033 & 0.0 & 0.0 \\
\hline 9 & C9 & 1.736 & 4.0 & 0.029 & 0.013 & 3.0 & 0.0 \\
\hline 10 & C10 & 1.595 & 4.0 & 0.029 & 0.059 & 3.0 & 0.0 \\
\hline 11 & C11 & 0.907 & -1.039 & 0.0590 & 0.059 & 0.0 & 0.0 \\
\hline 12 & C12 & -0.097 & 4.0 & 0.059 & 0.324 & 1.0 & 0.0 \\
\hline 13 & C13 & 0.768 & -1.713 & 0.059 & 0.006 & 0.0 & 0.0 \\
\hline 14 & C14 & -4.409 & -0.154 & 0.485 & 0.026 & 0.0 & 1.0 \\
\hline
\end{tabular}

C1 - Vitamin B12 (cobalamin); C2 - Vitamin H (Biotin); C3 - Folic acid (Vitamin M/Vitamin B9); C4 - Vitamin B1 (Thiamin); C5 - L-Carnitine (Vitamin BT);

C6 - Poly-N-vinylpyrrolidone; C7; Inulin; C8 - Poly cysteine; C9 - Chitosan; C10 - Pectin; C11 - Poly (propylene glycol); C12 - Poly (propylene imine);

C13 - Poly (lactic-co-glycolic acid); C14 - Deoxycholic acid 


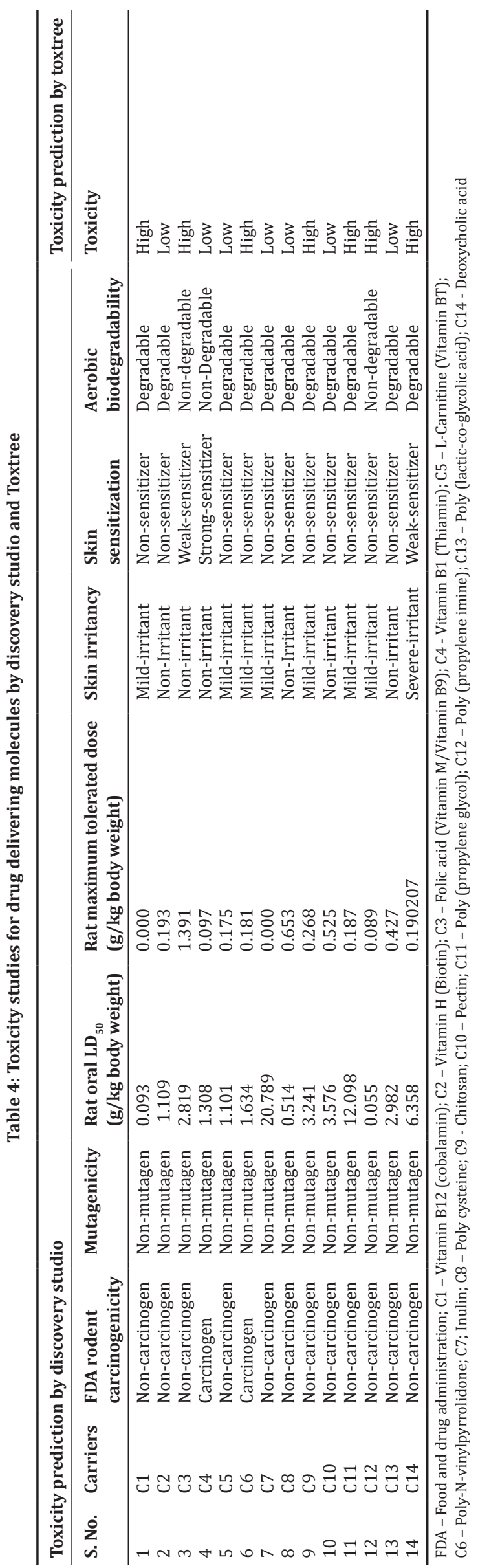

delivery process. Compare with other DDMs, inulin, Vitamin B12, and deoxycholic acid contains maximum hydrophobic regions (Table 2) that are responsible for efficient intestinal transport. The significant criteria, pharmacophoric active principle is more in folic acid, Vitamin B1, inulin, chitosan and pectin, but Vitamin B12 and poly(propylene imine) do not hold any single active principle in their structure. The analysis of pharmacophoric features suggests that Vitamin B1, inulin, chitosan, and pectin are possible DDMs for insulin.

\section{Analysis of physicochemical properties}

ADME descriptors of DDMs were evaluated by Discovery Studio and listed in Table 3 (Fig. 2). Vitamin H, Vitamin B1, poly cysteine, poly(propylene glycol), poly (lactic-co-glycolic acid), and deoxycholic acid are falls within 95\% absorption ellipse that shows efficient absorption (level = 0) across intestinal epithelium, concurrently shows the sign of maximum bioavailability in drug delivering mechanism. Poly-N-vinylpyrrolidone and poly(propylene imine) are falls within $99 \%$ absorption ellipse that shows moderate absorption (level $=1$ ); remaining compounds are falls outside 99\% absorption ellipse shows poor absorption (level = 3). In the case of aqueous solubility, folic acid (-3.378) shows extremely high aqueous solubility and drug-likeness. Vitamin H $(-1.432)$, Vitamin B1 $(-1.335)$, Poly- $\mathrm{N}$-vinylpyrrolidone $(-0.550)$, and poly(propylene imine) $(-0.097)$ show optimal aqueous solubility and drug-likeness; and others shows poor drug-likeness. In the point of BBB penetration, deoxycholic acid $(-0.154)$ is medium penetrant across BBB, because it is fall within 99\% confidence ellipsoids (level $=2$ ), the brain-blood ratio is between $0.3: 1$ and 1:1. Vitamin H (-1.229), Vitamin B1 $(-1.253)$, poly cysteine $(-1.362)$, poly(propylene glycol) $(-1.039)$, and poly (lactic-co-glycolic acid) $(-1.713)$ are low penetrants across BBB, because it is within $99 \%$ confidence ellipsoids (level $=3$ ), the brain-blood ratio is $<0.3: 1$. Other carriers are poor penetrants, because they are outside the $95 \%$ and $99 \%$ confidence ellipsoids (undefined level $=4$ ). In the case of CYP-4502D6 binding, the carrier should be non-inhibitor, because CYP-4502D6 is responsible for metabolism and elimination of drug molecules. All the listed DDMs are non-inhibitors, because their ADMET CYP2D6 Probability is $<0.5$. In the case of hepatotoxicity, most of the selected carriers are non-toxic because, their ADMET hepatotoxicity probability is $<0.5$. However, Vitamin B12 (0.509) and folic acid $(0.662)$ are toxic because, their ADMET hepatotoxicity probability is $>0.5$. Plasma protein binding capability should be $<90 \%$, then only the unbound molecule can easily penetrate the tissues to reach the active site and then to get eliminate. The plasma protein binding character of the all listed carriers is low and satisfies the standard value $(<4.0)$. Based on the overall results of physicochemical properties, Vitamin H, Vitamin B1, and deoxycholic acid are superior DDMs according to their efficient solubility in liquid, moderate penetration across BBB, enhanced absorption across intestinal epithelial cells, non-inhibition of CYP-4502D6 binding, unbound nature with plasma proteins and low hepatotoxicity.

\section{Analysis of toxicity}

Toxic scale is the most significant prediction for carriers in drug delivery mechanism. Toxicity properties such as FDA rodent carcinogenicity, mutagenicity, rat oral $\mathrm{LD}_{50}(\mathrm{~g} / \mathrm{kg}$ body weight), rat MTD $(\mathrm{g} / \mathrm{kg}$ body weight), skin irritancy, skin sensitization, aerobic biodegradability, and general toxicity were studied by discovery studio and Toxtree (Table 4 and Figs. 3 and 4). The listed carriers are non-mutagen and are noncarcinogen except Vitamin B1 and poly- $\mathrm{N}$-vinylpyrrolidone. The predicted skin irritancy is severe for deoxycholic acid, while Vitamin $\mathrm{H}$, folic acid, Vitamin B1, poly cysteine, pectin, and poly (lactic-co-glycolic acid) are non-irritants and others are in the category of mild-irritant. Vitamin B1 is predicted under strong skin-sensitizer, whereas folic acid and deoxycholic acid are weak skin-sensitizers; and others are falls under non skinsensitizer. The DDMs should be biodegradable in aerobic environment after delivering the drug to receptors; otherwise, it may leads to untoward reactivity. In this case, folic acid, Vitamin B1, and poly(propylene imine) are non-degradable, others are degradable under aerobic condition. The TOPKAT model predicts the rat oral acute median lethal dose $\left(\mathrm{LD}_{50}\right)$ in the toxicity test; and the rat MTD of all DDMs. According to general toxicity 
predition by Toxtree tool, Vitamin B12, folic acid, poly-N-vinylpyrrolidone, chitosan, poly(propylene glycol), poly(propylene imine), and deoxycholic acid may highly toxic while others are low in toxic category. Based on the analysis of toxicity studies, we concluded that Vitamin H, inulin, poly cysteine, pectin, and poly (lactic-co-glycolic acid) are safe carriers for delivering oral insulin molecule.

OICs

Designing

Human insulin monomer protein data bank (PDB ID: 1HLS), human insulin hexamer (PDB ID: 1AIO), and insulin lispro (PDB ID: 1 LPH) were retrieved from PDB and conjugated with all carriers individually. Kavimandan et al. [16] and Hinds et al. [17] suggest that conjugation of carriers with B1Phe, B27Thr, B28Pro, and B29Lys amino acids of human insulin and B28Lys amino acid of insulin lispro will be the efficient conjugate against ADMET barriers in oral delivery. Based on our computational analysis (discovery studio - LibDock), the positive LibDock score indicating the competent oral-insulin conjugation.

Human insulin monomer (PDB ID: 1HLS) conjugated individually with all listed DDMs (Table 5 and Fig. 5); among those, inulin was mono-conjugated efficiently with B1Phe of insulin monomer, and poly (lactic-co-glycolic acid)
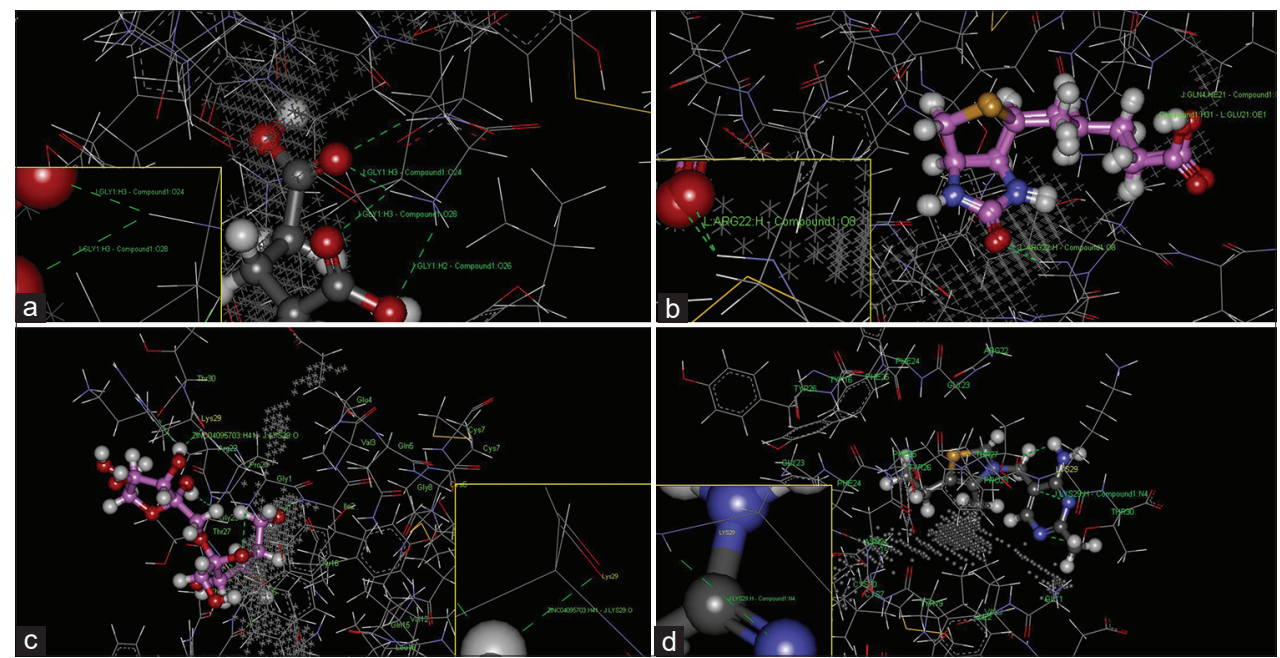

Fig. 3: (a) Bioconjugate from human insulin hexamer protein data bank (PDB ID: 1AIO) and Vitamin M is illustrated by discovery studio software. Vitamin M conjugated at GLY A1 and THR B27 amino acids of insulin hexamer. The inner figure illustrates the GLY 1 amino acid of insulin-A chain conjugates with Vitamin M. (b) Bioconjugate from human insulin hexamer (PDB ID: 1AIO) and Vitamin H is illustrated by discovery studio software. Vitamin H conjugated at GLN B4 and ARG B22 amino acids of insulin hexamer. The inner figure illustrates the ARG 22 amino acid of insulin-B chain conjugates with Vitamin H. (c) Bioconjugate from human insulin hexamer (PDB ID: 1AIO) and inulin is illustrated by discovery studio software. Inulin conjugated at LYS B29 amino acid of insulin hexamer. The inner figure illustrates

the LYS 29 amino acid of insulin-B chain conjugates with Inulin. (d) Bioconjugate from human insulin hexamer (PDB ID: 1AIO) and Vitamin B1 is illustrated by discovery studio software. Vitamin B1 conjugated at LYS B29 amino acid of insulin hexamer. The inner figure illustrates the LYS 29 amino acid of insulin-B chain conjugates with Vitamin B1

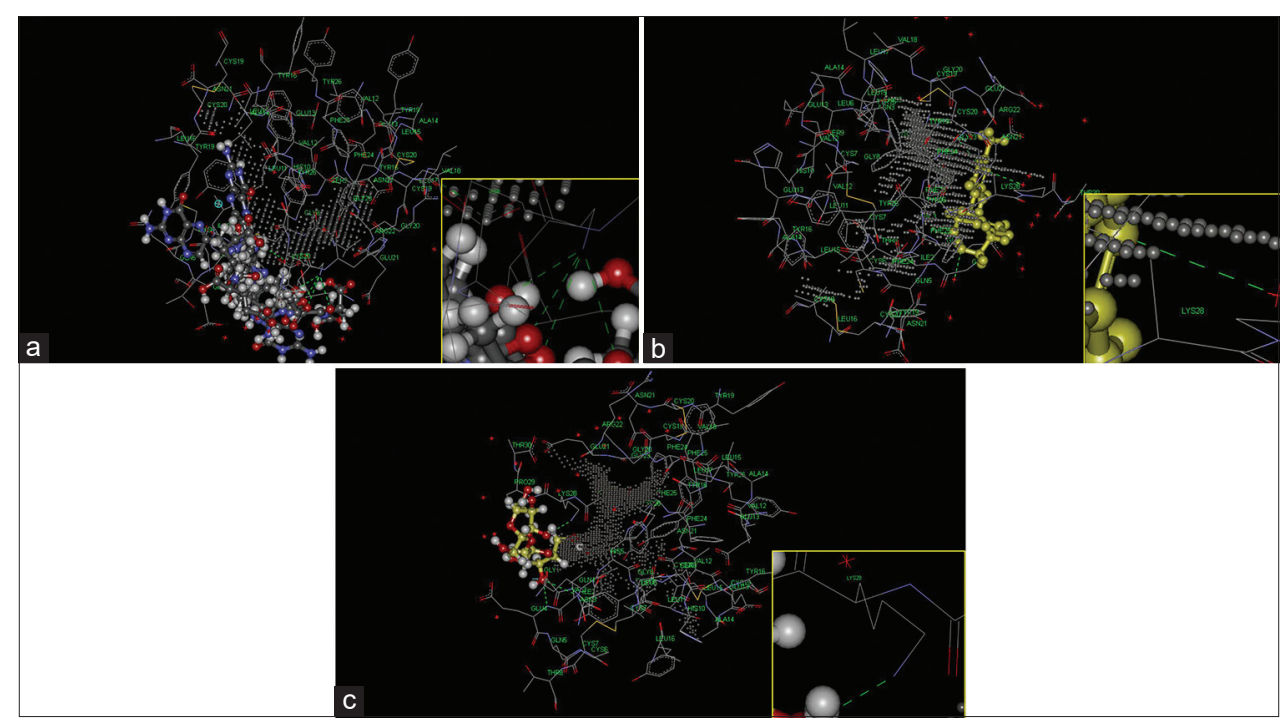

Fig. 4: (a) Bioconjugate from insulin lispro (PDB ID: 1 LPH) and Vitamin M is illustrated by discovery studio software. Vitamin M conjugated at GLY A1, ILE A2, VAL A3, GLU A4, TYR A19, GLN B4, HIS B5, THR B27, LYS B28, and THR B30 amino acids of insulin Lispro. The inner figure illustrates the LYS 28 amino acid of insulin-B chain conjugates with Vitamin M. (b) Bioconjugate from insulin lispro (PDB ID: 1 LPH) and Vitamin B1 is illustrated by discovery studio software. Vitamin B1 conjugated at GLU A4 and LYS B28 amino acids of insulin lispro. The inner figure illustrates the LYS 28 amino acid of insulin-B chain conjugates with Vitamin B1. (c) Bioconjugate from insulin lispro (PDB ID: 1 LPH) and inulin is illustrated by discovery studio software. Inulin conjugated at GLY A1, VAL A3, GLU A4, and LYS B28 amino acids of insulin lispro. The inner figure illustrates the LYS 28 amino acid of insulin-B chain conjugates with inulin 

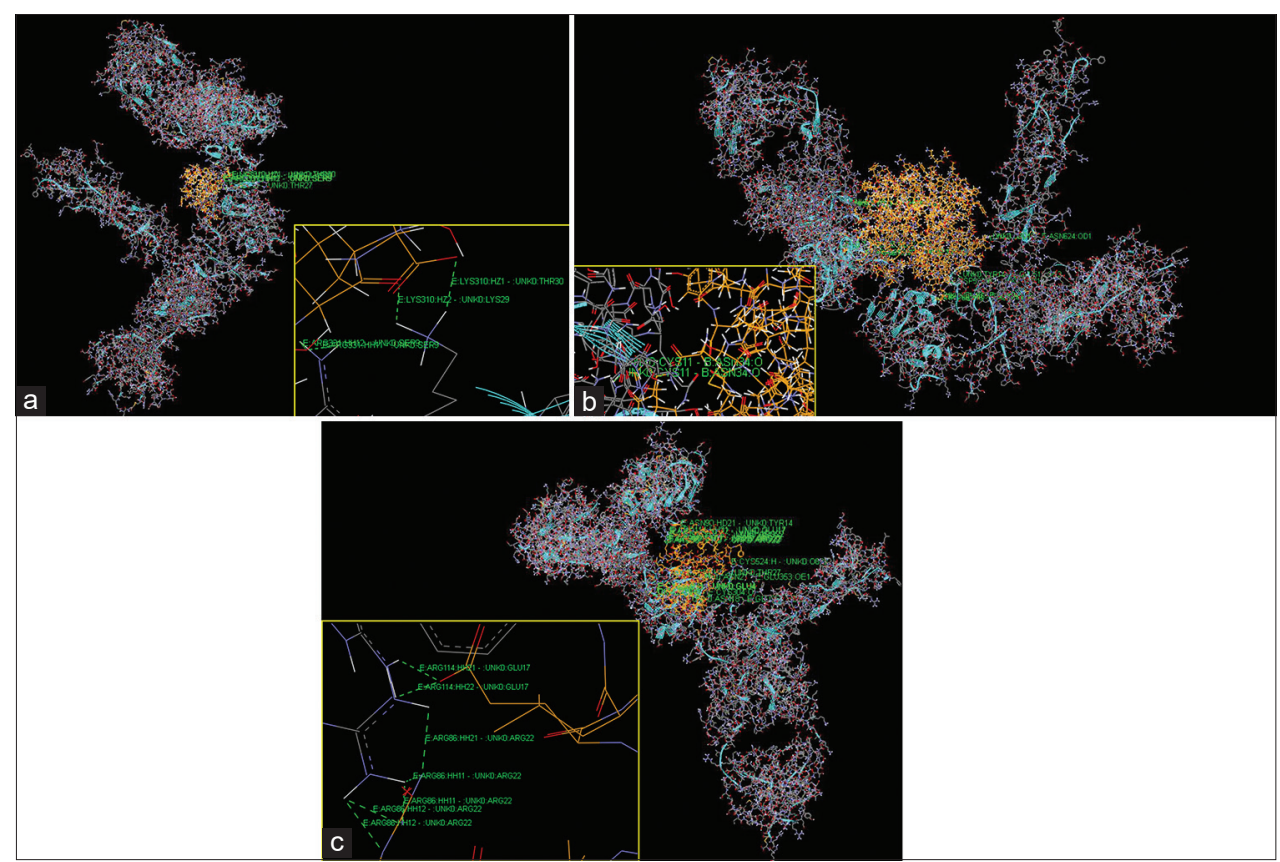

Fig. 5: (a) Interaction results of oral insulin conjugates (insulin monomer [1HLS] - drug delivering molecules [DDM] conjugates) with insulin receptor (IR). It does not show any interaction in leucine-rich repeat domain (L1, and residues 1-157) and in C-terminus of the $\alpha$-chain ( $\alpha$ CT, residues 704-715). The inner figure shows the interaction in LYS310 amino acid of IR which is not responsible for initiation of therapeutic effect. (b) Interaction results of oral insulin conjugates (insulin hexamer [1AIO]-DDM Conjugates) - DDM conjugates) with IR. It shows the interaction in ARG86, ASN34 of leucine-rich repeat domain (L1, residues 1-157) and no interaction in C-terminus of the $\alpha$-chain ( $\alpha \mathrm{CT}$, residues 704-715). The inner figure shows the interaction in ASN34 amino acid of IR which is responsible for initiation of therapeutic effect. (c) Interaction results of oral insulin conjugates (insulin lispro [1LPH] - DDM conjugates) with IR. It shows the interaction in ARG86, ASN90, and ARG114 of leucine-rich repeat domain (L1, residues 1-157) and no interaction in C-terminus of the $\alpha$-chain ( $\alpha \mathrm{CT}$, residues 704-715). The inner figure shows the interaction in ARG86, ARG114 amino acids of IR which is responsible for initiation of therapeutic effect

Table 5: Conjugation results of human insulin monomer (protein data bank ID: 1HLS), with all listed drug delivering molecules individually

\begin{tabular}{llllllll}
\hline S. No. & Conjugate & $\begin{array}{l}\text { Binding } \\
\text { site (LYS B29) }\end{array}$ & $\begin{array}{l}\text { Lip dock } \\
\text { score- }\end{array}$ & $\begin{array}{l}\text { Binding } \\
\text { site (PHE B1) }\end{array}$ & $\begin{array}{l}\text { Lip dock } \\
\text { score- }\end{array}$ & Other binding site & Lip dock score \\
\hline C1 & Vitamin B12 & - & - & - & - & - & \\
C2 & Vitamin H & - & - & - & - & CYS A11 HIS B10 GLN B4 & 86.2835 \\
C3 & Vitamin M & - & - & - & - & - & - \\
C4 & Vitamin B1 & - & - & - & - & CYS A6 CYS A11 LEU B6 & 94.1144 \\
C5 & Vitamin BT & - & - & - & - & LEU A13 VAL B2 & 68.0592 \\
C6 & poly-N-vinylpyrrolidone & - & - & - & - & - \\
C7 & Inulin & - & - & PHE B1 & 117.663 & - & - \\
C8 & Poly cysteine & - & - & - & GLN B4 & 53.1597 \\
C9 & Chitosan & - & - & - & CYS A11, VAL B2, ASN B3 & 74.4251 \\
C10 & Pectin & - & - & - & - & CYS A11 & 63.0902 \\
C11 & Poly (propylene glycol) & - & - & - & - & VAL B2 & 76.0238 \\
C12 & Poly (propylene imine) & - & - & PHE B1 & - & - \\
C13 & Poly (lactic-co-glycolic acid) & - & - & - & - & - & 68.6737 \\
C14 & Deoxycholic acid & - & CYS A11 & - \\
\hline
\end{tabular}

was di-conjugated competently with B1Phe and A11Cys of insulin monomer. Vitamin B12, Vitamin M, poly-N-vinylpyrrolidone, poly(propylene imine), and deoxycholic acid did not show any conjugation with amino acids of insulin monomer. Rest of the DDMs form the incompetent mono and diconjugates with A and B chain amino acids of insulin monomer. Based on the binding site (PHE B1) and LibDock score, inulin (117.663) shows the competent oral-insulin conjugation (Fig. 2c). Based on the LibDock score, Vitamin H (86.2835) and Vitamin B1 (94.1144) show the competent oralinsulin conjugation (Fig. 2a and b); but in the case of Poly (lactic-co-glycolic acid), the score is poor; even it conjugate at PHE B1 (Fig. 2d).

Human insulin hexamer (PDB ID: 1AIO) conjugated individually with all listed DDMs (Table 6 and Fig. 6); among those, Vitamin B1 and inulin were mono-conjugated efficiently with B29Lys of insulin hexamer and form the competent mono conjugates. Vitamin B12, poly-N-vinylpyrrolidone, and poly(propylene imine) did not show any conjugation with amino acids of insulin hexamer. Rest of the DDMs forms the incompetent mono and di-conjugates with A, B, C, D, E, F, G, H, I, J, K, and L chain amino acids of insulin hexamer. According to LibDock score, Vitamin M (114.324), Vitamin H (103.231), and inulin (94.3543) show the competent oral-insulin conjugation (Fig. 3a-c). Based on the binding site (LYS B29), inulin and Vitamin B1 show the competent oralinsulin conjugation (Fig. 3c and d).

Insulin lispro (PDB ID: $1 \mathrm{LPH}$ ) conjugated individually with all listed DDMs (Table 7 and Fig. 7); among those, Vitamin M was poly 
conjugated with $\mathrm{A}$ and $\mathrm{B}$ chain amino acids of insulin Lispro such as A1Gly, A2Ile, A3Val, A4Glu, A19Tyr, B4Gln, B5His, B27Thr, B28Lys, and B30Thr. This conjugates efficiently bonding with B27Thr and B28Lys amino acids. It also made the inefficient multiple bonding interactions with insulin Lispro and may affect the absorption and bioavailability of insulin Lispro. Vitamin B1 was di-conjugated reasonably with Lys B28 and Glu A4 of insulin Lispro. Inulin was tetra-conjugated fairly with B28Lys, A1Gly, A3Val, and A4Glu of insulin Lispro. Vitamin B12, Vitamin H, poly-N-vinylpyrrolidone, poly(propylene imine), and deoxycholic acid did not show any conjugation with amino acids of insulin Lispro. Rest of the DDMs forms the incompetent mono, di, tetra, and poly-conjugates with A and B chain amino acids of insulin Lispro. According to LibDock score and binding site (LYS B28), Vitamin M (131.57), Vitamin B1 (89.8971), and inulin (76.2195) show the competent oral-insulin conjugation (Fig. 4a-c).
Interaction of OICs with IR

Among the designed conjugates, inulin-insulin monomer conjugate, Vitamin B1-insulin monomer conjugate, Vitamin H-insulin monomer conjugate, Vitamin M-insulin hexamer conjugate, Vitamin H-insulin hexamer conjugate, inulin-insulin hexamer conjugate, Vitamin M-insulin lispro conjugate, Vitamin B1-insulin lispro conjugate, and inulin-insulin lispro conjugate are selected as capable OICs to interact with insulin receptor. In the case of insulin receptor, the leucine-rich repeat domain (L1, residues 1-157) and C-terminus of the $\alpha$-chain $(\alpha \mathrm{CT}$, residues 704-715) are Insulin binding surface and they functions as a signaling element to activate its tyrosine kinase and predicted to influence Insulin receptor-OIC interaction. In the proposed work, none of OICs shows interaction with $\alpha \mathrm{CT}$ while insulin monomer DDM conjugates (Fig. 5a) does not shows interaction with L1 domain (Table 8 and Fig. 5). Insulin hexamer - DDM conjugates interacts with ARG86 and ASN34 residues of IR (Fig. 5b), while insulin lispro - DDM

Table 6: Conjugation results of human insulin hexamer (protein data bank ID: 1AI0), with all listed drug delivering molecules individually

\begin{tabular}{|c|c|c|c|c|c|c|c|}
\hline S. No. & Conjugate & $\begin{array}{l}\text { Binding site } \\
\text { (LYS B29) }\end{array}$ & $\begin{array}{l}\text { Lip dock } \\
\text { score - }\end{array}$ & $\begin{array}{l}\text { Binding site } \\
\text { (PHE B1) }\end{array}$ & $\begin{array}{l}\text { Lip dock } \\
\text { score- }\end{array}$ & Other binding site & Lip dock score \\
\hline $\mathrm{C} 1$ & Vitamin B12 & - & - & - & - & - & - \\
\hline $\mathrm{C} 2$ & Vitamin $\mathrm{H}$ & - & - & - & - & GLN B4 ARG B22 & 103.231 \\
\hline $\mathrm{C} 3$ & Vitamin M & - & - & - & - & GLY A1 THR B27 & 114.324 \\
\hline $\mathrm{C} 4$ & Vitamin B1 & LYS B29 & 79.8834 & - & - & - & - \\
\hline $\mathrm{C} 5$ & Vitamin BT & - & - & - & - & ARG B22 & 84.7767 \\
\hline C6 & Poly-N-vinylpyrrolidone & - & - & - & - & - & - \\
\hline $\mathrm{C} 8$ & Poly cysteine & - & - & - & - & TYR B16 GLU B21 & 58.5629 \\
\hline $\mathrm{C} 9$ & Chitosan & - & - & - & - & TYR B16 TYR B26 & 90.016 \\
\hline C10 & Pectin & - & - & - & - & GLN B4 & 91.0549 \\
\hline C11 & Poly (propylene glycol) & - & - & - & - & GLU B21 ARG B22 & 71.5126 \\
\hline C12 & Poly (propylene imine) & - & - & - & - & - & - \\
\hline C13 & Poly (lactic-co-glycolic acid) & - & - & - & - & GLY B20 ARG B22 & 82.2602 \\
\hline C14 & Deoxycholic acid & - & - & - & - & GLY A1 ILE A2 & 60.763 \\
\hline
\end{tabular}

Table 7: Conjugation results of insulin lispro (protein data bank ID: 1 LPH), with all listed drug delivering molecules individually

\begin{tabular}{|c|c|c|c|c|c|c|c|}
\hline S. No. & Conjugate & $\begin{array}{l}\text { Binding } \\
\text { site (LYS B28) }\end{array}$ & $\begin{array}{l}\text { Lip dock } \\
\text { score- }\end{array}$ & $\begin{array}{l}\text { Binding } \\
\text { site (PHE B1) }\end{array}$ & $\begin{array}{l}\text { Lip } \\
\text { dock } \\
\text { score- }\end{array}$ & Other binding site & $\begin{array}{l}\text { Lip dock } \\
\text { score }\end{array}$ \\
\hline $\mathrm{C} 1$ & Vitamin B12 & - & - & - & - & - & - \\
\hline $\mathrm{C} 2$ & Vitamin $\mathrm{H}$ & - & - & - & - & - & - \\
\hline $\mathrm{C} 3$ & Vitamin M & LYS B28 & 131.57 & - & - & $\begin{array}{l}\text { GLY A1 ILE A2, VAL A3, GLU A4, TYR } \\
\text { A19, GLN B4 HIS B5 THR B27, THR B30 }\end{array}$ & 131.57 \\
\hline $\mathrm{C} 4$ & Vitamin B1 & LYS B28 & 89.8971 & - & - & GLU A4 & 89.8971 \\
\hline C6 & Poly-N-vinylpyrrolidone & - & - & - & - & - & - \\
\hline $\mathrm{C} 7$ & Inulin & LYS B28 & 76.2195 & - & - & GLY A1 VAL A3, GLU A4 & 76.2195 \\
\hline $\mathrm{C} 8$ & Poly cysteine & - & - & - & - & GLN B4 GLU A4 & 48.858 \\
\hline C9 & Chitosan & - & - & - & - & GLY A1 ILE A2 GLU A4 THR B27 & 67.1639 \\
\hline $\mathrm{C} 10$ & Pectin & - & - & - & - & GLY A1 GLU A4 & 73.2077 \\
\hline C11 & Poly (propylene glycol) & - & - & - & - & GLY A1 & 57.6819 \\
\hline $\mathrm{C} 12$ & Poly (propylene imine) & - & - & - & - & - & - \\
\hline C13 & Poly (lactic-co-glycolic acid) & - & - & - & - & $\begin{array}{l}\text { GLY A1, ILE A2, GLU A4, VAL A3, THR } \\
\text { B27, GLN } 44\end{array}$ & 64.8442 \\
\hline C14 & Deoxycholic acid & - & - & - & - & - & - \\
\hline
\end{tabular}

Table 8: Interaction results of oral insulin conjugates with insulin receptor

\begin{tabular}{llll}
\hline S. No. & Conjugates & $\begin{array}{l}\text { Interaction with leucine-rich repeat } \\
\text { domain (L1, residues 1-157) }\end{array}$ & $\begin{array}{l}\text { Interaction with C-terminus of the } \\
\boldsymbol{\alpha} \text {-chain ( } \boldsymbol{\alpha C T} \text {, residues 704-715) }\end{array}$ \\
\hline 1 & Insulin monomer (1HLS) - DDM conjugates & No interaction & No interaction \\
2 & Insulin hexamer (1AIO) - DDM conjugates & ARG86 ASN34 & No interaction \\
3 & Insulin lispro (1LPH) - DDM conjugates & ARG86 ASN90 ARG114 & No interaction \\
\hline
\end{tabular}


conjugates interacts with ARG86, ASN90, and ARG114 residues of IR (Fig. 5c) which reflects the efficient binding affinity of insulin lispro DDM conjugates with IR.

\section{DISCUSSION}

In our analysis, 14 DDMs were screened and its characteristic features for oral delivery of insulin were examined. Based on the toxicity and conjugation ability with insulin, the DDMs were chosen for developmental studies. The overall results are summarized below:

Vitamin B12 is chemically inert; may cause Vitamin-B12 deficiency by prolonged dose regimen [18] by experimental text-mining. It shows poor pharmacophoric and hepatotoxic features; non-carcinogen but highly toxic as per drug informatics analysis. It did not show any conjugation with insulin monomer, insulin hexamer, and insulin Lispro. Vitamin B12 is not recommended for in vitro and in vivo studies.

Vitamin $\mathrm{H}$ is inert; may suppress the biological activity of drug by experimental text-mining. It shows poor pharmacophoric and efficient ADME features; it is non-carcinogen, non-mutagen, and low toxic as per drug informatics analysis. It shows efficient conjugation with insulin monomer, insulin hexamer, and not with insulin Lispro. Vitamin $\mathrm{H}$-insulin hexamer conjugate alone shows efficient interaction with IR. Vitamin H is not recommended for further developmental studies.

Vitamin $M$ does not produce any untoward reactivity during drugdelivery mechanism [19] based on text-mining; it shows poor pharmacophoric and hepatotoxic features; it is highly toxic as per drug informatics analysis. It shows efficient conjugation with insulin hexamer, insulin lispro, and not with insulin monomer. Vitamin M-insulin hexamer and Vitamin M- insulin Lispro conjugates show efficient interaction with IR. Vitamin M is an optional molecule for further developmental studies.

Vitamin B1 completely satisfy the defined criteria based on bio-text mining; it shows better pharmacophoric and efficient ADME features; it is carcinogen but low toxic as per drug informatics analysis; it shows efficient conjugation with insulin monomer, insulin hexamer, and insulin Lispro. Vitamin B1-insulin hexamer and Vitamin B1-insulin Lispro conjugates show efficient interaction with IR. Vitamin B1 is a highly recommended molecule for in vitro and in vivo studies.

Vitamin BT partially satisfy the defined criteria based on bio-text mining; it shows poor pharmacophoric and moderate ADME features; it is low toxic as per drug informatics analysis. It shows poor conjugation with insulin monomer, insulin hexamer, and insulin lispro. Vitamin BT is not recommended for further developmental studies.

Poly-N-vinylpyrrolidone is partially fulfilling the defined criteria based on bio-text mining. It shows poor pharmacophoric and moderate ADME features; it is carcinogen and highly toxic as per drug informatics analysis. It does not show any conjugation with insulin monomer, insulin hexamer, and insulin Lispro. Poly- $\mathrm{N}$-vinylpyrrolidone is not recommended for further developmental studies.

Inulin absolutely satisfy the defined criteria based on bio-text mining; it shows superior pharmacophoric and moderate ADME features; it is non-carcinogen, non-mutagen, and low toxic as per drug informatics analysis. It shows efficient conjugation with insulin monomer, insulin hexamer, and insulin lispro. Inulin-insulin hexamer and inulin-insulin lispro conjugates show efficient interaction with IR. Inulin is a highly recommended molecule for in vitro and in vivo studies.

Poly cysteine is partially fulfill the defined criteria based on bio-text mining; it shows poor pharmacophoric and moderate ADME features; it is less toxic as per drug informatics analysis. It did not show any efficient conjugation with insulin monomer, insulin hexamer, and insulin lispro. Poly cysteine is not suggested for in vitro and in vivo studies.
Chitosan completely satisfy the defined criteria based on bio-text mining; it shows better pharmacophoric and moderate ADME features; it is highly toxic as per drug informatics analysis. It did not show any efficient conjugation with insulin monomer, insulin hexamer, and insulin lispro. Chitosan is not suggested for in vitro and in vivo studies.

Pectin is partially fulfill the defined criteria based on bio-text mining; it shows superior pharmacophoric and moderate ADME features; meantime it is non-carcinogen, non-mutagen, and low toxic as per drug informatics analysis; meantime it did not show any efficient conjugation with insulin monomer, insulin hexamer, and insulin Lispro. Pectin is not suggested for in-vitro and in-vivo studies.

Poly (propylene glycol) partially fulfill the defined criteria based on bio-text mining; it shows poor pharmacophoric and moderate ADME features; it is non-carcinogen, non-mutagen, but highly toxic as per drug informatics analysis. It did not show any efficient conjugation with insulin monomer, insulin hexamer, and insulin lispro. Poly (propylene glycol) is not suggested for in vitro and in vivo studies. In earlier studies, propylene glycol showed toxic symptoms after the frequent doses and repeated application when used as a vehicle in medicinal preparations [20]. In drug delivering mechanisms, it eliminates the toxic degradation [21], and less toxic than the parent substance (poly ethylene glycol) [22], because PEG is a frequently used DDM for oral insulin delivery. Thus poly (propylene glycol) may be a possible carrier for further studies.

Poly (propylene imine) is partially fulfill the defined criteria based on bio-text mining; it shows poor pharmacophoric and moderate ADME features; it is non-carcinogen, non-mutagen, but highly toxic as per drug informatics analysis. It did not show any conjugation with insulin monomer, insulin hexamer, and insulin lispro. Poly (propylene imine) is not suggested for in vitro and in vivo studies.

Poly (lactic-co-glycolic acid) fulfills the defined criteria based on bio-text mining; it shows poor pharmacophoric and moderate ADME features; it is non-carcinogen, non-mutagen, and low toxic as per drug informatics analysis. It shows efficient conjugation with insulin monomer and not with insulin hexamer and insulin lispro. Poly (lactic-co-glycolic acid)insulin monomer conjugate does not shows any interaction with IR. Poly (lactic-co-glycolic acid) is not an optional molecule for the further developmental studies.

Deoxycholic acid partially fulfilling the defined criteria based on bio-text mining; it shows moderate pharmacophoric and efficient ADME features; it is highly toxic as per drug informatics analysis. It did not show any efficient conjugation with insulin monomer, insulin hexamer, and insulin lispro. Deoxycholic acid is not suggested for in vitro and in vivo studies.

While compare with the binding efficiency of insulin molecules; insulin lispro (LysB29) shows the competent conjugation with DDMs and the resultant conjugates show therapeutically capable interaction with IR and correlate with insulin monomeric and hexameric form of conjugates.

\section{CONCLUSION}

The oral bioavailability of insulin is $1 \%$. It may be enhanced by novel carriers that deliver insulin to the site of absorption. In the proposed work, based on the defined criteria 14 DDMs were filtered from seven reputed compound databases and its Pharmacophoric and ADMET properties were analyzed by biomedical text mining and drug-informatic tools. The results from the conducted studies concluded that inulin and Vitamin B1 are the novel, safe, and proficient oral carriers for insulin. (Poly ethylene glycol) is an optional vehicle for oral delivery of insulin. Insulin lispro is the tremendous option for oral delivery than other insulin forms. Clinical studies are recommended to develop our results.

\section{ACKNOWLEDGMENT}

The author thanks management of St. Joseph's College, Bengaluru, for supporting this work. 


\section{AUTHOR CONTRIBUTION}

The author has carried out all the work individually.

\section{CONFLICTS OF INTEREST}

No known conflicts of interest.

\section{AUTHORS FUNDING}

No funding support for this research work.

\section{REFERENCES}

1. Cockburn IM, Henderson RM. Scale and scope in drug development: Unpacking the advantages of size in pharmaceutical research. J Health Econ 2001;20:1033-57.

2. Gowthamarajan K, Kulkarni GT. Oral insulin-fact or fiction? Resonance 2003;8:38-46.

3. Clement S, Still JG, Kosutic G, McAllister RG. Oral insulin product hexyl-insulin monoconjugate $2\left(\mathrm{HIM}_{2}\right)$ in Type 1 diabetes mellitus: The glucose stabilization effects of $\mathrm{HIM}_{2}$. Diabetes Technol Ther 2002;4:459-66.

4. Wearley LL. Recent progress in protein and peptide delivery by noninvasive routes. Crit Rev Ther Drug Carrier Syst 1991;8:331-94.

5. Lee S, Kim K, Kumar TS, Lee J, Kim SK, Lee DY, et al. Synthesis and biological properties of insulin-deoxycholic acid chemical conjugates. Bioconjug Chem 2005;16:615-20.

6. Macedo A, Filipe P, Thomé NG, Vieira J, Oliveira C, Teodósio C, et al. A brief overview of the oral delivery of insulin as an alternative to the parenteral delivery. Curr Mol Med 2020;20:134-43.

7. Petrus AK, Vortherms AR, Fairchild TJ, Doyle RP. Vitamin B12 as a carrier for the oral delivery of insulin. Chem Med Chem 2007;2:1717-21.

8. Murray-Rust J, McLeod AN, Blundell TL, Wood SP. Structure and evolution of insulins: Implications for receptor binding. Bioessays 1992; $14: 325-31$

9. Jeffrey PD. The self-association of zinc-free bovine insulin. Four model patterns and their significance. Biol Chem Hoppe Seyler 1990;371:1165-74.
10. Uchio T, Baudyš M, Liu F, Song SC, Kim SW. Site-specific insulin conjugates with enhanced stability and extended action profile. Adv Drug Deliv Rev 1999;35:289-306.

11. Oprea TI, Davis AM, Teague SJ, Leeson PD. Is there a difference between leads and drugs? A historical perspective. J Chem Inf Comput Sci 2001;41:1308-15.

12. Zhu X, Ji J, Huang D, Zhu Y, Tang C, Yang X, et al. Discovery, synthesis and evaluation of novel cholesterol absorption inhibitors. Chem Biol Drug Des 2012;80:426-33.

13. Rybacka A, Rudén C, Andersson PL. On the use of in silico tools for prioritising toxicity testing of the low-volume industrial chemicals in REACH. Basic Clin Pharmacol Toxicol 2014;115:77-87.

14. Ravi L, Ragunathan A. Potential drug targets for Aloin and microdontin: An in-silico analysis. Asian J Pharm Clin Res 2016;9:194-6.

15. Kim S, Thiessen PA, Bolton EE, Chen J, Fu G, Gindulyte A, et al. PubChem substance and compound databases. Nucleic Acids Res 2016;44:D1202-13.

16. Kavimandan NJ, Losi E, Wilson JJ, Brodbelt JS, Peppas NA. Synthesis and characterization of insulin-transferrin conjugates. Bioconjug Chem 2006; 17:1376-84.

17. Hinds K, Koh JJ, Joss L, Liu F, Baudyš M, Kim SW. Synthesis and characterization of poly(ethylene glycol)-insulin conjugates. Bioconjug Chem 2000;11:195-201.

18. Deegan KL, Jones KM, Zuleta C, Ramirez-Zea M, Lildballe DL, Nexo E, et al. Breast milk Vitamin B-12 concentrations in Guatemalan women are correlated with maternal but not infant Vitamin B-12 status at 12 months postpartum. J Nutr 2012;142:112-6.

19. Yan L, Chen W, Zhu X, Huang L, Wang Z, Zhu G, et al. Folic acid conjugated self-assembled layered double hydroxide nanoparticles for high-efficacy-targeted drug delivery. Chem Commun 2013;49:10938-40.

20. Martin G, Finberg L. Propylene glycol: A potentially toxic vehicle in liquid dosage form. J Pediatr 1970;77:877-8.

21. Runge MB, Wang H, Spinner RJ, Windebank AJ, Yaszemski MJ. Reformulating polycaprolactone fumarate to eliminate toxic diethylene glycol: Effects of polymeric branching and autoclave sterilization on material properties. Acta Biomater 2012;8:133-43.

22. Inchem I. Chemical safety information from intergovernmental organizations. Can Cent 2011. 